\title{
What Healthcare Workers Should Know about Environmental Bacterial Contamination in the Intensive Care Unit
}

\author{
Vincenzo Russotto, ${ }^{1}$ Andrea Cortegiani, ${ }^{1}$ Teresa Fasciana, ${ }^{2}$ Pasquale Iozzo, ${ }^{1}$ \\ Santi Maurizio Raineri, ${ }^{1}$ Cesare Gregoretti, ${ }^{1}$ Anna Giammanco, ${ }^{2}$ and Antonino Giarratano ${ }^{1}$ \\ ${ }^{1}$ Department of Biopathology and Medical Biotechnologies (DIBIMED), Section of Anesthesia, Analgesia, \\ Intensive Care and Emergency, University Hospital Policlinico Paolo Giaccone, University of Palermo, Palermo, Italy \\ ${ }^{2}$ Department of Sciences for Health Promotion and Mother and Child Care, University of Palermo, Palermo, Italy \\ Correspondence should be addressed to Vincenzo Russotto; vinrussotto@gmail.com
}

Received 31 March 2017; Revised 7 September 2017; Accepted 28 September 2017; Published 29 October 2017

Academic Editor: Paul M. Tulkens

Copyright (C) 2017 Vincenzo Russotto et al. This is an open access article distributed under the Creative Commons Attribution License, which permits unrestricted use, distribution, and reproduction in any medium, provided the original work is properly cited.

\begin{abstract}
Intensive care unit- (ICU-) acquired infections are a major health problem worldwide. Inanimate surfaces and equipment contamination may play a role in cross-transmission of pathogens and subsequent patient colonization or infection. Bacteria contaminate inanimate surfaces and equipment of the patient zone and healthcare area, generating a reservoir of potential pathogens, including multidrug resistant species. Traditional terminal cleaning methods have limitations. Indeed patients who receive a bed from prior patient carrying bacteria are exposed to an increased risk (odds ratio 2.13, 95\% confidence intervals 1.62-2.81) of being colonized and potentially infected by the same bacterial species of the previous patient. Biofilm formation, even on dry surfaces, may play a role in reducing the efficacy of terminal cleaning procedures since it enables bacteria to survive in the environment for a long period and provides increased resistance to commonly used disinfectants. No-touch methods (e.g., UV-light, hydrogen peroxide vapour) are under investigation and further studies with patient-centred outcomes are needed, before considering them the standard of terminal cleaning in ICUs. Healthcare workers should be aware of the role of environmental contamination in the ICU and consider it in the broader perspective of infection control measures and stewardship initiatives.
\end{abstract}

\section{Introduction}

Intensive care unit- (ICU-) acquired infections are a major health problem worldwide. Emergence of multidrug resistant organisms (MDROs) poses a daily challenge to ICU physicians, dealing with critically ill patients with multiple risk factors for infections (i.e., impairment of body barriers due to invasive devices and surgery, immunosuppression, prolonged antibiotic exposure) [1-4]. A relevant body of evidence highlights the high prevalence of contamination of hightouch surfaces and equipment surrounding patients' bed [5]. Indeed, the patient's nearby environment is crowded by equipment for monitoring and organ support (e.g., monitors, ventilator, extracorporeal life support machines), requiring sophisticated and specific cleaning procedures. Contamination of inanimate surfaces may occur as the consequence of direct patient shedding of bacteria (higher from infected than colonized patients) or via healthcare workers' (HCWs') hands. HCWs contaminate their hands from inanimate surfaces as frequently as direct patient contact [6]. In a randomized cross-over study, recontamination of high-touch surfaces in ICU occurred after only 4 hours from standard cleaning measures [7]. Environmental contamination in the ICU involves not only equipment for direct patient care (e.g., stethoscopes, ultrasound equipment, surfaces of mechanical ventilators) but also surfaces of objects used for clinical data recordings (i.e., medical charts, computer keyboard, and mouse) and mobile phones [8]. Environmental contamination has been identified as a major contributor of bacteria cross-transmission and patient colonization and infection. In 1991, Weinstein [9] estimated the relative contribution of different potential sources for ICU-acquired infections: 40-60\% patient's endogenous flora, followed by cross-infection via HCWs' hands (20-40\%), antibiotic-driven changes in flora 


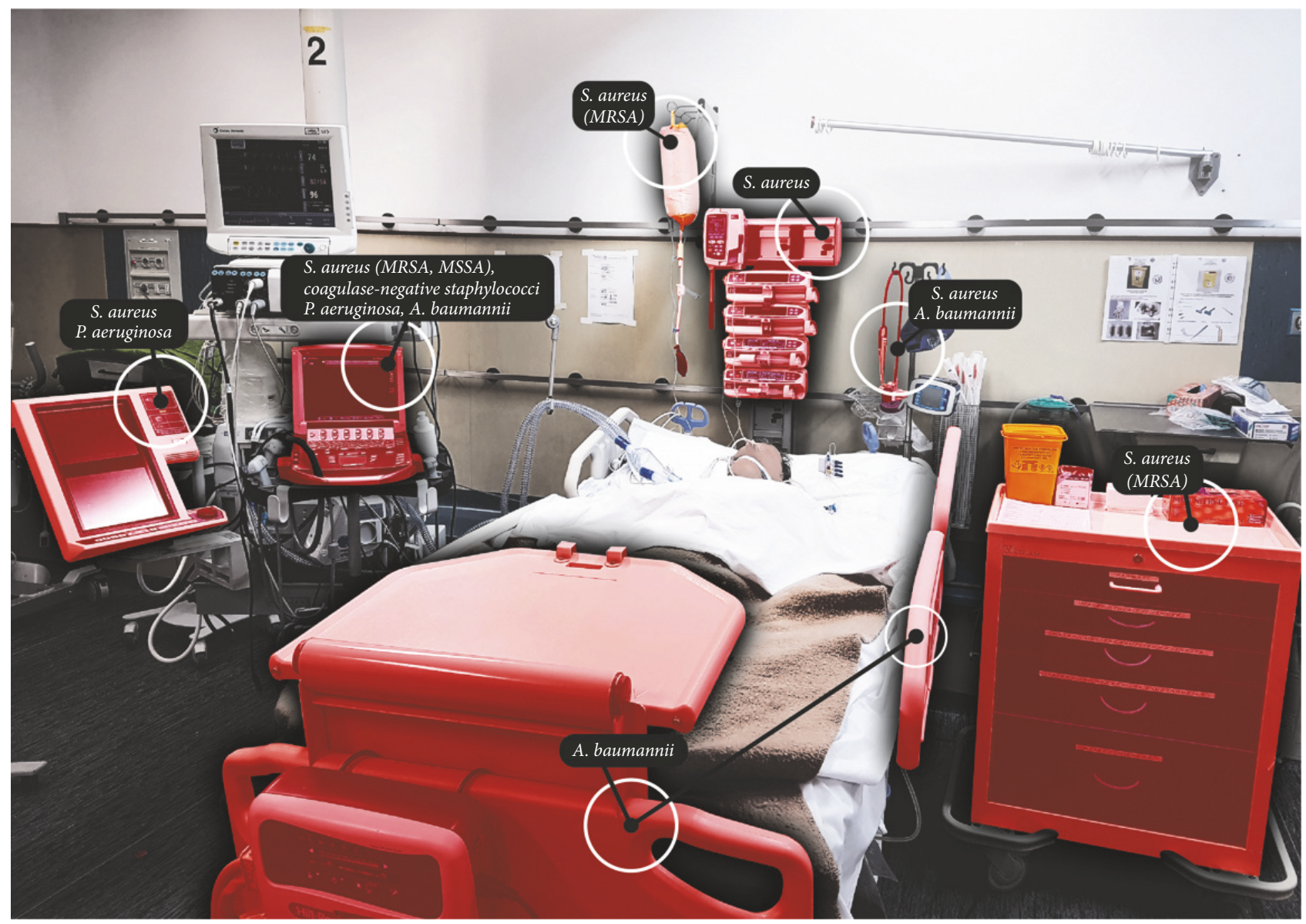

FIGURE 1: Patient zone with more frequently isolated bacteria contaminating inanimate surfaces and equipment.

(20-25\%), and other sources (including environmental contamination, 20\%). Understanding the mechanisms underlying cross-transmission of pathogens from inanimate surfaces and equipment may contribute to lay the foundation of effective infection control measures aiming to halt the spread of healthcare-associated infections. The aim of this review is to provide updated evidence on environmental contamination in the ICU, focusing on mechanisms by which bacteria are able to survive on inanimate surfaces, describing the concept of patient zone and healthcare area and the role of contamination for ICU-acquired colonization and infection.

1.1. The Concepts of Patient Zone and Healthcare Area. The concepts of patient zone and healthcare area have been introduced as a user-centred, operative behaviour aiming to enhance hand hygiene compliance [10]. The patient zone encompasses the patient and surfaces and equipment surrounding him/her (i.e., bed rails, ventilator, monitors). The healthcare area is composed of all surfaces outside a given patient zone (i.e., the healthcare facility environment and other patient zones) [8].

The healthcare area may be contaminated by bacteria from different patient zones. Inanimate surfaces in the patient zone are contaminated by bacteria colonizing/infecting patients in two ways: direct shedding from patients and via
HCWs' hands. High-touch objects in the immediate vicinity of patients are heavily contaminated. A higher degree and rate of contamination occur from infected patients than from patients who are only colonized. Moreover, a correlation exists between number of culture-positive body sites and environmental contamination [11, 12]. A high degree of patient zone contamination has been reported also in case of patients with diarrhea $[13,14]$.

Figure 1 shows a patient zone with most frequently reported contaminating bacteria in the literature.

\subsection{ICU-Acquired Colonization and Infection: Update on} Available Evidence. Evidence on the role of environmental contamination for cross-transmission of pathogens comes from studies reporting on outbreaks of infections driven by contaminated objects or equipment, studies investigating the association of colonized/infected patients with environmental and HCWs' hands contamination, and studies reporting on the risk of acquiring bacteria from prior bed occupants [15]. Hand-washing sinks, bottled still water, and bronchoscope suction valves have been related to outbreaks registered in ICUs, with the same strains and antibiotic susceptibility profiles registered in those isolated from colonized/infected patients [16-21]. This observation is of value when we 
consider the role of inanimate surfaces contamination as a reservoir of MDROs of potentially pathogen role.

In their cohort study, Morgan et al. [22] investigated how frequently HCWs contaminated gloves and gowns after contact with patients. Approximately, after one of every three interactions with a patient carrying Acinetobacter baumannii, HCWs contaminated their gloves and gowns. A. baumannii was present in almost $80 \%$ of rooms from colonized patients. Contamination with A. baumannii occurred more frequently than with other bacteria (Pseudomonas aeruginosa, vancomycin-resistant Enterococci and methicillinresistant Staphylococcus aureus). Independent risk factors for HCWs contamination by MDROs were positive environmental cultures (OR 4.2, 95\% CI 2.7-6.5), stay in room for more than 5 minutes (OR 2.0, 95\% CI 1.2-3.4), performing physical examination (OR 1.7, 95\% CI 1.1-2.8), and contact with the ventilator (OR 1.8, 95\% CI 1.1-2.8) [22].

A number of studies reported on a higher risk of acquiring bacteria from prior room occupants. This independent risk factor occurred for both Gram-positive (S. aureus, Enterococcus species, Clostridium difficile) and Gram-negative bacteria (Acinetobacter spp., P. aeruginosa, Klebsiella pneumoniae) [23], including MDROs (MRSA, VRE). We recently performed a meta-analysis of studies investigating this issue in the ICU setting [24]. The pooled OR of acquisition of bacteria from prior bed occupants was 2.13 (95\% CI 1.62-2.81). When we considered the OR for the acquisition according to bacterial species, we registered the highest OR for A. baumannii (OR 4.91, 95\% CI 2.79-8.64) and C. difficile (OR 2.57, 95\% CI 1.28-5.15). It is remarkable that this increased risk occurs even when current terminal cleaning procedures are addressed. We may speculate that these findings may be explained by suboptimal terminal cleaning procedures resulting in persisting surfaces contamination. Environmental contamination may represent the reservoir for cross-transmission of bacteria via HCWs' hands. Structural ICU features may be associated with a different degree of environmental contamination and cross-transmission rate. Indeed, single-room ICUs have the theoretical advantage of a physical separation of different patient zones. This may be also associated with the ease of adoption of enhanced terminal cleaning procedures requiring environment isolation. However, cross-transmission of bacteria from prior bed occupant occurred in single-room ICUs in most studies [25].

1.3. Terminal Cleaning in ICU. The term terminal cleaning refers to all methods used for disinfection of either a room or a patient zone between occupying patients (i.e., after patient discharge). Quaternary ammonium and bleach are the most commonly used products for this purpose. The efficacy of terminal cleaning relies on different factors, including training and management of personnel (e.g., adequate contact time, compliance with protocols) and accessibility of surfaces. If we consider the higher patient's risk of acquiring a MDRO if exposed to the bed of a previously colonized/infected patient, current terminal cleaning methods are far from being considered a highly effective procedure. Inadequate cleaning as assessed by objective measures of operators' performance has been reported in different studies. Programs including a training phase, followed by an objective monitoring of operators' performance through the use of assays such as adenosine triphosphate (ATP) bioluminescence, resulted in an overall improvement of performance and reduced degree of environmental contamination [26, 27]. In a prospective study [28], authors investigated a bundle of interventions aiming to enhance room cleaning in ICUs after patient discharge. They collected baseline cleaning performance using a mark visible only with an ultraviolet lamp (blacklight indicator). Study interventions consisted of an increased disinfectant volume application, an educational campaign involving the cleaning staff and the adoption of a black-light monitoring system for feedback. The indicator was removed from $72 \%$ of surfaces after the interventions, compared to $44 \%$ cleaned surfaces at baseline $(p<0.001)$. The interventions also reduced the environmental positive cultures for MRSA and VRE [28]. Similar results were reported in another prospective study conducted in 27 ICUs where only $49.5 \%$ of selected surfaces were cleaned at baseline, compared to $82 \%$ after a structured educational, procedural, administrative intervention and objective feedback provided by a fluorescent marker [29].

In 2010 the Centres for Disease Control and Prevention provided a toolkit with a bundle of interventions for improvement of hospital cleaning. Objective assessment has been incorporated among the interventions to apply to enhance cleaning performance. The proposed methods were direct cleaning practice observation, swab and agar slide cultures, fluorescent markers, and ATP bioluminescence [30]. Recently, no-touch terminal cleaning procedures have been investigated in ICUs. They consist of the use of either physical (e.g., ultraviolet, UV-light) or chemical agents (e.g., hydrogen peroxide, $\mathrm{H}_{2} \mathrm{O}_{2}$ vapour, HPV) delivered by specific devices without the active role of personnel. The theoretical advantages of these techniques are a higher overall performance given the capability to disinfect difficult to reach surfaces, difficult to clean equipment, and the lack of reliance on personnel performance.

UV-light generates DNA and RNA alterations leading to an irreversible damage and killing of microorganisms. The device is normally placed in the room centre and it is activated by remote control. Surfaces receiving direct irradiation are exposed to the highest killing potential but wall, floor, and other surfaces are able to reflect the UV-light and indirectly expose other surfaces. However, shadowed areas may not receive a sufficient radiation dose and UV-light does not penetrate porous surfaces. Two different UV-power levels are available for killing of vegetative and spore-forming pathogens. Different studies reported the high capacity of UV radiation to significantly reduce the contamination of high-touch surfaces by a number of pathogens (MRSA, VRE and C. difficile) [31, 32]. Compared to HPV, UV-light decontamination is cheaper to operate and maintain and requires less time [33]. Anderson et al. [34] recently performed a large (31226 exposed patients) multicentre randomized study investigating the adoption of enhanced terminal cleaning procedures of seed rooms by previously colonized/infected patients. They compared 4 different strategies: UV-light combined with quaternary ammonium, UV-light combined 
with bleach, bleach, and reference (quaternary ammonium). Notably, according to their standard cleaning protocol, bleach was used as reference for rooms seed by $C$. difficile. Adding UV-light led to a reduction of the incidence of colonization and infection caused by MRSA and VRE, while authors did not observe a statistically significant difference when UVlight was compared to bleach. Of note, only one outcome occurred for Acinetobacter and therefore the role of enhanced terminal cleaning was not investigated for this pathogen. The lack of benefit for $C$. difficile cross-transmission was explained by the adoption of an enhanced procedure also in the reference group (i.e., bleach), the high compliance of personnel to the cleaning protocol (which may be significantly lower in real life), and the use of a single-stage cycle of UV-light for a pathogen with a time- and dose-dependent response to UVlight. Despite these limitations, this is the first trial to date enrolling such a high number of patients and adopting the clinically relevant outcomes of infections and colonization [35].

$\mathrm{H}_{2} \mathrm{O}_{2}$ is a noncorrosive agent showing bactericidal, fungicidal, sporicidal, and virucidal properties in vitro. $\mathrm{H}_{2} \mathrm{O}_{2}$ damages lipid membranes, DNA, and RNA through its oxidative action. HPV showed effective decontamination against MRSA, VRE, Acinetobacter spp., K. pneumonia, and C. difficile spores [36]. $\mathrm{H}_{2} \mathrm{O}_{2}$ may be released in three different forms: dry vapour, wet vapour, and mist. These three technologies produce $\mathrm{H}_{2} \mathrm{O}_{2}$ particles small enough to diffuse and reach hidden and difficult to reach surfaces. Recently, Blazejewski et al. [37] investigated the efficiency of HPV in improving disinfection in ICUs. They applied HPV after routine terminal cleaning and collected environmental sampling before and after HPV use. After patient discharge, $8 \%$ of sampled rooms were contaminated with at least $1 \mathrm{MDRO}$. Routine terminal cleaning reduced the environmental bacterial load but authors did not detect a statistically significant difference in the degree of MDROs contamination [37]. HPV, instead, significantly reduced the residual environmental contamination by MDROs. Given the high costs for implementation of no-touch terminal cleaning methods in ICUs (i.e., machines and maintenance costs, additional staff members), further studies are needed to evaluate their impact for patient-centred outcomes.

\section{How Bacteria Survive on Inanimate Surfaces and after Terminal Cleaning Procedures?}

The principal factors associated with the ability of a nosocomial pathogen to survive on inanimate surfaces and equipment are the specific microorganism characteristics (such as genus, species, specific strain, ability to form biofilm, and microorganism concentration) and the environmental factors (such as UV radiation, temperature, humidity, presence of organic materials, and surface type) [38-40]. Evidence on the capacity to survive in environmental reservoirs has been reported for bacteria ( $C$. difficile, VRE, MRSA, $P$. aeruginosa, Escherichia coli, Klebsiella spp., and Acinetobacter spp.), viruses (influenza, parainfluenza, enteric, hepatitis B viruses), and fungi (Candida albicans, Candida glabrata, Candida parapsilosis, Aspergillus spp., and Zygomycetes) [41-47].
Microorganisms are able to survive on surfaces because of their production of adhesion molecules and biofilms. These abilities are favoured when microorganisms grow on materials with high absorptive capacity [48]. Coagulase-negative staphylococci are able to survive up to 8-21 days on cotton used to produce clothing and towels, while $P$. aeruginosa survives for only 2-24 hours on the same surface. Even different species from the same genus showed different survival capacity [42]. As an example, C. parapsilosis showed higher resistance when compared to C. albicans or C. krusei [49]. Intrinsic microbiologic features also influence the resistance against disinfectants. For example, mycobacteria have a waxy cell wall able to prevent disinfectants entry, whereas Gramnegative bacteria have an outer membrane acting as a barrier preventing the uptake of disinfectants [50]. Concentration of Gram-positive and Gram-negative bacteria, fungi, and viruses may influence their persistence on surfaces; the greater the microbial load, the longer the survival capacity. A biofilm is a structured community of microorganisms encased and attached to surfaces by exopolymeric substances (EPS). Up to $90 \%$ of biofilm are made of EPS, which provides protection against environmental desiccation. Biofilm plays an important role in catheter-related infections and of other indwelling medical devices [51]. Bacteria are able to form biofilm also on dry inanimate surfaces. It has been speculated that biofilm formation may be enhanced by a thin film of water resulting from condensation on surfaces or that the relative humidity of ICUs is sufficiently high to allow biofilm formation [52]. Biofilms contain a high bacterial load able to survive on dry hospital surfaces for a long time, showing also an increased resistance towards inactivation by disinfectants. Indeed, bacteria in the biofilm are up to 1000-fold more resistant to disinfectants than their corresponding planktonic form $[40,52-55]$. P. aeruginosa biofilm on flexible endoscopes surfaces is able to survive 5-minute treatment with peracetic acid at 2000 parts per million concentration, which is the working concentration used by some washer-disinfectors [54].

Vickery et al. [52] investigated the persistence of reservoirs of MDROs within biofilm after terminal cleaning in an ICU. Equipment and furnishings were aseptically removed from the ICU, scanned by electron microscopy and cultured. Biofilm was demonstrated on 4 of the five different samples from the patient zone and healthcare area. Cultures from samples led to MRSA growth. This finding highlights one possible explanation of the suboptimal terminal cleaning efficacy and the persistence of a reservoir of MDROs possibly involved in direct or indirect (via HCWs' hands) crosstransmission [52]. This should be considered in the broader perspective of emergence of MDROs, stewardship initiatives, and infection control measures [45, 56-58].

The increased resistance of biofilms to disinfectants is supposed to be due to the following factors:

(i) gene regulation of microorganisms with increased lateral gene transfer and mutation rates [59];

(ii) phenotypic adaptation of cells to sublethal disinfectant concentration [60]; 
(iii) production of EPS surrounding the bacteria. The EPS reduces the penetration of biocides into the biofilm, inactivates some disinfectants by binding to them, and inactivates some disinfectants by liberation of enzymes [61];

(iv) biofilm which can be composed by different microorganism species constituting a polymicrobial biofilm with a higher resistance to disinfectants compared to monospecies biofilms [61]. The mechanism of this increased resistance may result from an increased disinfectant inactivation due to a more complex EPS or from shielding of sensitive organisms by externally situated disinfectant tolerant organisms [62].

Among environmental variables, ultraviolet (UV) light, temperature, humidity, and presence of organic material have been reported to have a major role in influencing microbial viability. Visible light and UV radiation are generally deleterious to microorganisms. Temperatures higher than $50^{\circ} \mathrm{C}$ are able to kill most Candida spp. while low temperatures $\left(4^{\circ} \mathrm{C}\right.$ to $\left.6^{\circ} \mathrm{C}\right)$ increase survival times for many bacteria. Humidity can have variable effects on the persistence of microorganisms on surfaces. Yeast showed a better survive at higher humidity [42]. Organic matter (e.g., blood, serum, sputum, pus, fecal material) may play both a direct and an indirect role to enhance environmental resistance of microorganisms. The direct role is the barrier effect protecting microorganisms from environmental physical and chemical agents. The indirect role is the interference of organic matter with the antimicrobial activity of disinfectants through chemical reactions resulting in a complex exhibiting less germicidal or nongermicidal properties and leaving a reduced quantity of active disinfectant agents. This frequently occurs with chlorine and iodine disinfectants [63]. In parallel with development of new strategies to enhance disinfectant agents' efficacy, different research groups are now focusing on development of novel materials which may potentially be used to prevent or reduce contamination and biofilm formation by bacteria, including MDROs. Metal-embedded surfaces (copper, gallium, and titanium) were effective at preventing planktonic and biofilm growth of $P$ aeruginosa, $S$. aureus, and E. coli tested strains [64]. In a recently published observational study, silver-embedded screens used to separate ICU beds were more effective than traditional cloth screens at reducing surfaces contamination and crosstransmission of pathogens [65].

\section{Conclusions}

Inanimate surfaces and equipment contamination play a major role in cross-transmission of pathogens in ICUs. Bacteria, including MDROs, may survive for a long time to environmental physical and chemical agents and have been isolated from different surfaces and equipment of the patient zone and of the healthcare area [8]. Traditional terminal cleaning methods showed major flaws and no-touch methods are under investigation $[24,34]$. Clinicians should be aware of the issue of environmental contamination and consider it in the broader perspective of infection control measures and stewardship initiatives [58].

\section{Conflicts of Interest}

The authors declare no conflicts of interest.

\section{Acknowledgments}

The authors thank Roberto Di Fresco, freelance graphic designer, for his help in preparing the figures.

\section{References}

[1] V. Russotto, A. Cortegiani, G. Graziano et al., "Bloodstream infections in intensive care unit patients: distribution and antibiotic resistance of bacteria," Infection and Drug Resistance, vol. 8, pp. 287-296, 2015.

[2] A. Tabah, D. Koulenti, K. Laupland et al., "Characteristics and determinants of outcome of hospital-acquired bloodstream infections in intensive care units: The EUROBACT International Cohort Study," Intensive Care Medicine, vol. 38, no. 12, pp. 1930-1945, 2012.

[3] A. Cortegiani, V. Russotto, G. Graziano et al., "Use of cepheid xpert carba- $\mathrm{r}^{\circledR}$ for rapid detection of carbapenemase-producing bacteria in abdominal septic patients admitted to intensive care unit," PLoS ONE, vol. 11, no. 8, Article ID e0160643, 2016.

[4] Z. Yu, W. Qin, J. Lin, S. Fang, and J. Qiu, "Antibacterial mechanisms of polymyxin and bacterial resistance," BioMed Research International, vol. 2015, Article ID 679109, 11 pages, 2015.

[5] C. E. Adams, J. Smith, V. Watson, C. Robertson, and S. J. Dancer, "Examining the association between surface bioburden and frequently touched sites in intensive care," Journal of Hospital Infection, vol. 95, no. 1, pp. 76-80, 2017.

[6] M. K. Hayden, D. W. Blom, E. A. Lyle, C. G. Moore, and R. A. Weinstein, "Risk of hand or glove contamination after contact with patients colonized with vancomycin-resistant Enterococcus or the colonized patients' environment," Infection Control and Hospital Epidemiology, vol. 29, no. 2, pp. 149-154, 2008.

[7] A. P. R. Wilson, D. Smyth, G. Moore et al., "The impact of enhanced cleaning within the intensive care unit on contamination of the near-patient environment with hospital pathogens: A randomized crossover study in critical care units in two hospitals," Critical Care Medicine, vol. 39, no. 4, pp. 651-658, 2011.

[8] V. Russotto, A. Cortegiani, S. M. Raineri, and A. Giarratano, "Bacterial contamination of inanimate surfaces and equipment in the intensive care unit," Journal of Intensive Care, vol. 3, no. 1, 2015.

[9] R. A. Weinstein, "Epidemiology and control of nosocomial infections in adult intensive care units," American Journal of Medicine, vol. 91, no. 3, pp. S179-S184, 1991.

[10] H. Sax, B. Allegranzi, I. Uçkay, E. Larson, J. Boyce, and D. Pittet, "My five moments for hand hygiene': a user-centred design approach to understand, train, monitor and report hand hygiene," Journal of Hospital Infection, vol. 67, no. 1, pp. 9-21, 2007.

[11] U. Rohr, A. Kaminski, M. Wilhelm, L. Jurzik, S. Gatermann, and G. Muhr, "Colonization of patients and contamination of the patients' environment by MRSA under conditions of single-room isolation," International Journal of Hygiene and Environmental Health, vol. 212, no. 2, pp. 209-215, 2009. 
[12] M. J. M. Bonten, M. K. Hayden, C. Nathan et al., "Epidemiology of colonisation of patients and environment with vancomycinresistant enterococci," The Lancet, vol. 348, no. 9042, pp. 16151619, 1996.

[13] J. M. Boyce, N. L. Havill, J. A. Otter, and N. M. T. Adams, "Widespread environmental contamination associated with patients with diarrhea and methicillin-resistant Staphylococcus aureus colonization of the gastrointestinal tract," Infection Control and Hospital Epidemiology, vol. 28, no. 10, pp. 1142-1147, 2007.

[14] M. H. Samore, "Clinical and molecular epidemiology of sporadic and clustered cases of nosocomial Clostridium difficile diarrhea," American Journal of Medicine, vol. 100, no. 1, pp. 3240, 1996.

[15] D. J. Weber and W. A. Rutala, "Introduction: Understanding and preventing transmission of health careassociated pathogens due to the contaminated hospital environment," Infection Control and Hospital Epidemiology, vol. 34, no. 5, pp. 449-452, 2013.

[16] G. Aygun, O. Demirkiran, T. Utku et al., "Environmental contamination during a carbapenem-resistant Acinetobacter baumannii outbreak in an intensive care unit," Journal of Hospital Infection, vol. 52, no. 4, pp. 259-262, 2002.

[17] C. Lowe, B. Willey, A. O'Shaughnessy et al., "Outbreak of extended-spectrum $\beta$-lactamase-producing Klebsiella oxytoca infections associated with contaminated handwashing sinks," Emerging Infectious Diseases, vol. 18, no. 8, pp. 1242-1247, 2012.

[18] D. Roux, B. Aubier, H. Cochard, R. Quentin, and N. Van Der Mee-Marquet, "Contaminated sinks in intensive care units: An underestimated source of extended-spectrum beta-lactamaseproducing Enterobacteriaceae in the patient environment," Journal of Hospital Infection, vol. 85, no. 2, pp. 106-111, 2013.

[19] T. Eckmanns, M. Oppert, M. Martin et al., "An outbreak of hospital-acquired Pseudomonas aeruginosa infection caused by contaminated bottled water in intensive care units," Clinical Microbiology and Infection, vol. 14, no. 5, pp. 454-458, 2008.

[20] M. Guy, P. Vanhems, C. Dananché et al., "Outbreak of pulmonary Pseudomonas aeruginosa and Stenotrophomonas maltophilia infections related to contaminated bronchoscope suction valves, Lyon, France, 2014," European Communicable Disease Bulletin, vol. 21, no. 28, 2016.

[21] F. Salm, M. Deja, P. Gastmeier et al., "Prolonged outbreak of clonal MDR Pseudomonas aeruginosa on an intensive care unit: Contaminated sinks and contamination of ultra-filtrate bags as possible route of transmission?" Antimicrobial Resistance and Infection Control, vol. 5, no. 1, article no. 53, 2016.

[22] D. J. Morgan, E. Rogawski, K. A. Thom et al., "Transfer of multidrug-resistant bacteria to healthcare workers' gloves and gowns after patient contact increases with environmental contamination," Critical Care Medicine, vol. 40, no. 4, pp. 1045-1051, 2012.

[23] S. Nseir, C. Blazejewski, R. Lubret, F. Wallet, R. Courcol, and A. Durocher, "Risk of acquiring multidrug-resistant Gramnegative bacilli from prior room occupants in the intensive care unit," Clinical Microbiology and Infection, vol. 17, no. 8, pp. 12011208, 2011.

[24] V. Russotto, A. Cortegiani, S. M. Raineri, P. Iozzo, C. Gregoretti, and A. Giarratano, "What is the risk of acquiring bacteria from prior intensive care unit bed occupants?" Critical Care, vol. 21, no. 1, article no. 55, 2017.

[25] V. Russotto, A. Cortegiani, C. Gregoretti, S. M. Raineri, and A. Giarratano, "ICU-acquired infections: It is not only about the number of patients per room," Journal of Critical Care, vol. 34, p. 30, 2016.

[26] V. Russotto, A. Cortegiani, S. M. Raineri, C. Gregoretti, and A. Giarratano, "Adenosine triphosphate bioluminescence in intensive care units: Be careful with its use," American Journal of Infection Control, vol. 44, no. 6, pp. 732-733, 2016.

[27] P. Carling, "Methods for assessing the adequacy of practice and improving room disinfection," American Journal of Infection Control, vol. 41, no. 5, pp. S20-S25, 2013.

[28] E. R. Goodman, R. Platt, R. Bass, A. B. Onderdonk, D. S. Yokoe, and S. S. Huang, "Impact of an environmental cleaning intervention on the presence of methicillin-resistant Staphylococcus aureus and vancomycin-resistant enterococci on surfaces in intensive care unit rooms," Infection Control and Hospital Epidemiology, vol. 29, no. 7, pp. 593-599, 2008.

[29] P. C. Carling, M. F. Parry, L. A. Bruno-Murtha, and B. Dick, "Improving environmental hygiene in 27 intensive care units to decrease multidrug-resistant bacterial transmission," Critical Care Medicine, vol. 38, no. 4, pp. 1054-1059, 2010.

[30] A. Guh and P. Carling, Options for Evaluating Environmental Cleaning, Centers for Disease Control and Prevention, 2010.

[31] J. M. Boyce, N. L. Havill, and B. A. Moore, “Terminal decontamination of patient rooms using an automated mobile UV light unit," Infection Control and Hospital Epidemiology, vol. 32, no. 8, pp. 737-742, 2011.

[32] M. M. Nerandzic, J. L. Cadnum, M. J. Pultz, and C. J. Donskey, "Evaluation of an automated ultraviolet radiation device for decontamination of Clostridium difficile and other healthcareassociated pathogens in hospital rooms," BMC Infectious Diseases, vol. 10, article no. 197, 2010.

[33] C. Blazejewski, M.-J. Guerry, S. Preau, A. Durocher, and S. Nseir, "New methods to clean ICU rooms," Infectious Disorders - Drug Targets, vol. 11, no. 4, pp. 365-375, 2011.

[34] D. J. Anderson, L. F. Chen, D. J. Weber et al., "Enhanced terminal room disinfection and acquisition and infection caused by multidrug-resistant organisms and Clostridium difficile (the Benefits of Enhanced Terminal Room Disinfection study): a cluster-randomised, multicentre, crossover study," The Lancet, vol. 389, no. 10071, pp. 805-814, 2017.

[35] V. Russotto, A. Cortegiani, P. Iozzo, S. M. Raineri, C. Gregoretti, and A. Giarratano, "No-touch methods of terminal cleaning in the intensive care unit: results from the first large randomized trial with patient-centred outcomes," Critical Care, vol. 21, no. 1, 2017.

[36] J. A. Otter and G. L. French, "Survival of nosocomial bacteria and spores on surfaces and inactivation by hydrogen peroxide vapor," Journal of Clinical Microbiology, vol. 47, no. 1, pp. 205207, 2009.

[37] C. Blazejewski, F. Wallet, A. Rouzé et al., "Efficiency of hydrogen peroxide in improving disinfection of ICU rooms," Critical Care, vol. 19, no. 1, article no. 30, 2015.

[38] D. C. Esteves, V. C. Pereira, J. M. Souza et al., "Influence of biological fluids in bacterial viability on different hospital surfaces and fomites," American Journal of Infection Control, vol. 44, no. 3, pp. 311-314, 2016.

[39] S. Galvin, A. Dolan, O. Cahill, S. Daniels, and H. Humphreys, "Microbial monitoring of the hospital environment: why and how?" Journal of Hospital Infection, vol. 82, no. 3, pp. 143-151, 2012.

[40] A. C. Abreu, R. R. Tavares, A. Borges, F. Mergulhão, and M. Simões, "Current and emergent strategies for disinfection of 
hospital environments," Journal of Antimicrobial Chemotherapy, vol. 68, no. 12, Article ID dkt281, pp. 2718-2732, 2013.

[41] B. Hota, "Contamination, disinfection, and cross-colonization: are hospital surfaces reservoirs for nosocomial infection?" Clinical Infectious Diseases, vol. 39, no. 8, pp. 1182-1189, 2004.

[42] A. Kramer, I. Schwebke, and G. Kampf, "How long do nosocomial pathogens persist on inanimate surfaces? A systematic review," BMC Infectious Diseases, vol. 6, article 130, 2006.

[43] D. J. Weber, W. A. Rutala, M. B. Miller, K. Huslage, and E. Sickbert-Bennett, "Role of hospital surfaces in the transmission of emerging health care-associated pathogens: Norovirus, Clostridium difficile, and Acinetobacter species," American Journal of Infection Control, vol. 38, no. 5, pp. S25-S33, 2010.

[44] F. C. Lessa, C. V. Gould, and L. C. McDonald, "Current status of Clostridium difficile infection epidemiology," Clinical Infectious Diseases, vol. 55, supplement 2, pp. S65-S70, 2012.

[45] C. Mammina, D. M. Palma, C. Bonura et al., "Epidemiology and clonality of carbapenem-resistant Acinetobacter baumannii from an intensive care unit in Palermo, Italy," BMC Research Notes, vol. 5, article no. 365, 2012.

[46] P. Di Carlo, G. Pantuso, A. Cusimano et al., "Two cases of monomicrobial intraabdominal abscesses due to KPC - 3 Klebsiella pneumoniae ST258 clone," BMC Gastroenterology, vol. 11, article no. 103, 2011.

[47] A. Cortegiani, V. Russotto, A. Maggiore et al., "Antifungal agents for preventing fungal infections in non-neutropenic critically ill patients," Cochrane Database of Systematic Reviews, 2016.

[48] C. Cala, E. Amodio, E. Di Carlo, R. Virruso, T. Fasciana, and A. Giammanco, "Biofilm production in Staphylococcus epidermidis strains isolated from the skin of hospitalized patients: Genetic and phenotypic characteristics," New Microbiologica, vol. 38, no. 4, pp. 521-529, 2015.

[49] A. N. Neely and M. M. Orloff, "Survival of some medically important fungi on hospital fabrics and plastics," Journal of Clinical Microbiology, vol. 39, no. 9, pp. 3360-3361, 2001.

[50] A. D. Russell, "Bacterial resistance to disinfectants: Present knowledge and future problems," Journal of Hospital Infection, vol. 43, no. 4, pp. S57-S68, 1999.

[51] C. Beloin, N. Fernández-Hidalgo, and D. Lebeaux, "Understanding biofilm formation in intravascular device-related infections," Intensive Care Medicine, vol. 43, no. 3, pp. 443-446, 2017.

[52] K. Vickery, A. Deva, A. Jacombs, J. Allan, P. Valente, and I. B. Gosbell, "Presence of biofilm containing viable multiresistant organisms despite terminal cleaning on clinical surfaces in an intensive care unit," Journal of Hospital Infection, vol. 80, no. 1, pp. 52-55, 2012.

[53] J. A. Otter, K. Vickery, J. T. Walker et al., "Surface-attached cells, biofilms and biocide susceptibility: implications for hospital cleaning and disinfection," Journal of Hospital Infection, vol. 89, no. 1, pp. 16-27, 2015.

[54] A. Akinbobola, L. Sherry, W. Mckay, G. Ramage, and C. Williams, "Tolerance of Pseudomonas aeruginosa in in-vitro biofilms to high-level peracetic acid disinfection," Journal of Hospital Infection, vol. 97, no. 2, pp. 162-168, 2017.

[55] F. Gunther, B. Blessing, E. Tacconelli, and N. T. Mutters, "MRSA decolonization failure-are biofilms the missing link?" Antimicrobial Resistance and Infection Control, vol. 6, no. 1, article no. 32, 2017.

[56] P. C. Carling and J. M. Bartley, "Evaluating hygienic cleaning in health care settings: What you do not know can harm your patients," American Journal of Infection Control, vol. 38, no. 5, pp. S41-S50, 2010.

[57] A. Cortegiani, V. Russotto, P. Iozzo, S. M. Raineri, and A. Giarratano, "Rapid detection of carbapenem resistance: Targeting a zero level of inadequate empiric antibiotic exposure," Critical Care, vol. 20, no. 1, article no. 404, 2016.

[58] J. Garnacho Montero, F. Á. Lerma, P. R. Galleymore et al., "Combatting resistance in intensive care: The multimodal approach of the Spanish ICU “Zero Resistance” program," Critical Care, vol. 19, no. 1, article no. 114, 2015.

[59] E. Skippington and M. A. Ragan, "Lateral genetic transfer and the construction of genetic exchange communities," FEMS Microbiology Reviews, vol. 35, no. 5, pp. 707-735, 2011.

[60] C. A. Fux, J. W. Costerton, P. S. Stewart, and P. Stoodley, "Survival strategies of infectious biofilms," Trends in Microbiology, vol. 13, no. 1, pp. 34-40, 2005.

[61] A. Bridier, R. Briandet, V. Thomas, and F. Dubois-Brissonnet, "Resistance of bacterial biofilms to disinfectants: a review," Biofouling, vol. 27, no. 9, pp. 1017-1032, 2011.

[62] I. Machado, S. P. Lopes, A. M. Sousa, and M. O. Pereira, "Adaptive response of single and binary Pseudomonas aeruginosa and Escherichia coli biofilms to benzalkonium chloride," Journal of Basic Microbiology, vol. 52, no. 1, pp. 43-52, 2012.

[63] D. L. Lewis and M. Arens, "Resistance of microorganisms to disinfection in dental and medical devices," Nature Medicine, vol. 1, no. 9, pp. 956-958, 1995.

[64] N. Gugala, J. A. Lemire, and R. J. Turner, "The efficacy of different anti-microbial metals at preventing the formation of, and eradicating bacterial biofilms of pathogenic indicator strains," The Journal of Antibiotics, vol. 70, no. 6, pp. 775-780, 2017.

[65] J. Ruiz, P. Ramirez, E. Villarreal et al., "Silver-embedded screens in the intensive care unit. A new tool to control multi-drug resistant bacterial cross-transmission," European Journal of Clinical Microbiology and Infectious Diseases, vol. 36, no. 8, pp. 1505-1509, 2017. 


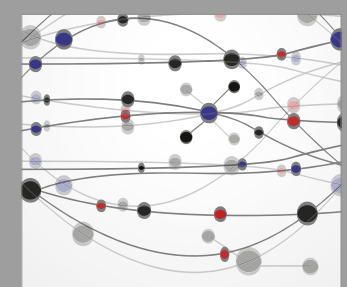

The Scientific World Journal
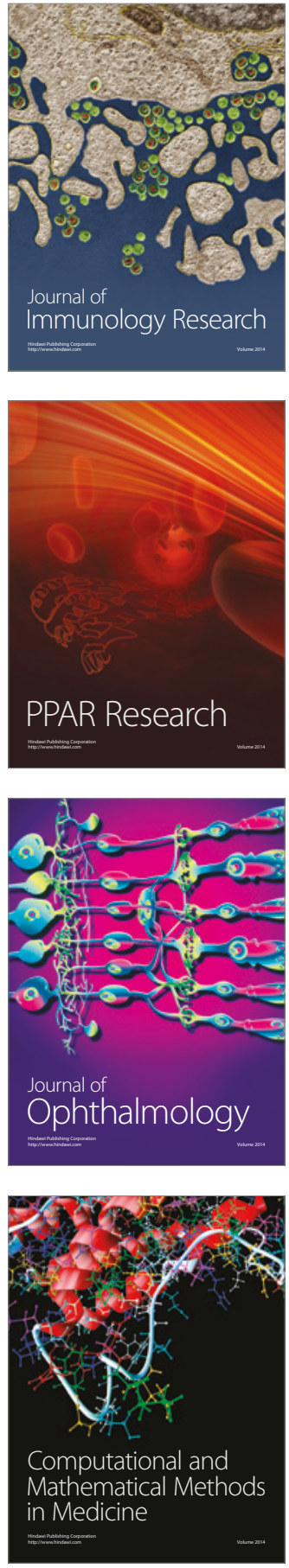

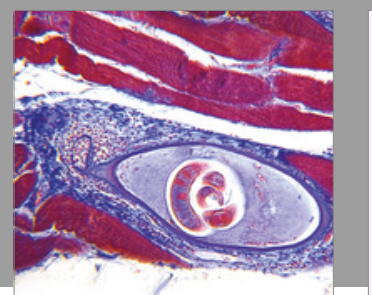

Gastroenterology Research and Practice
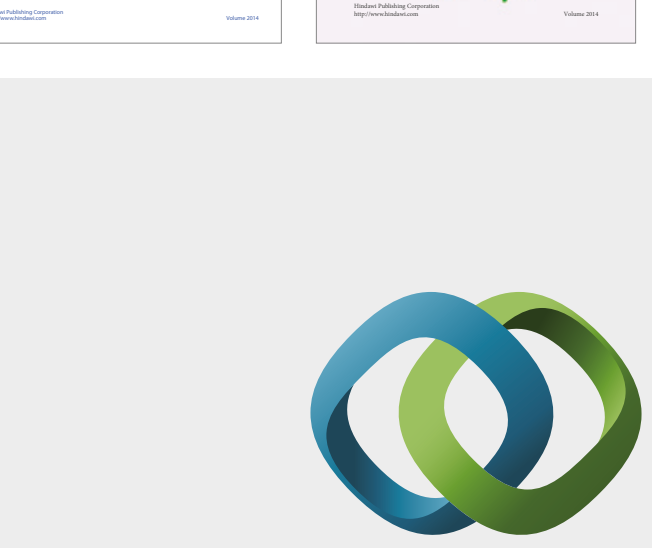

\section{Hindawi}

Submit your manuscripts at

https://www.hindawi.com
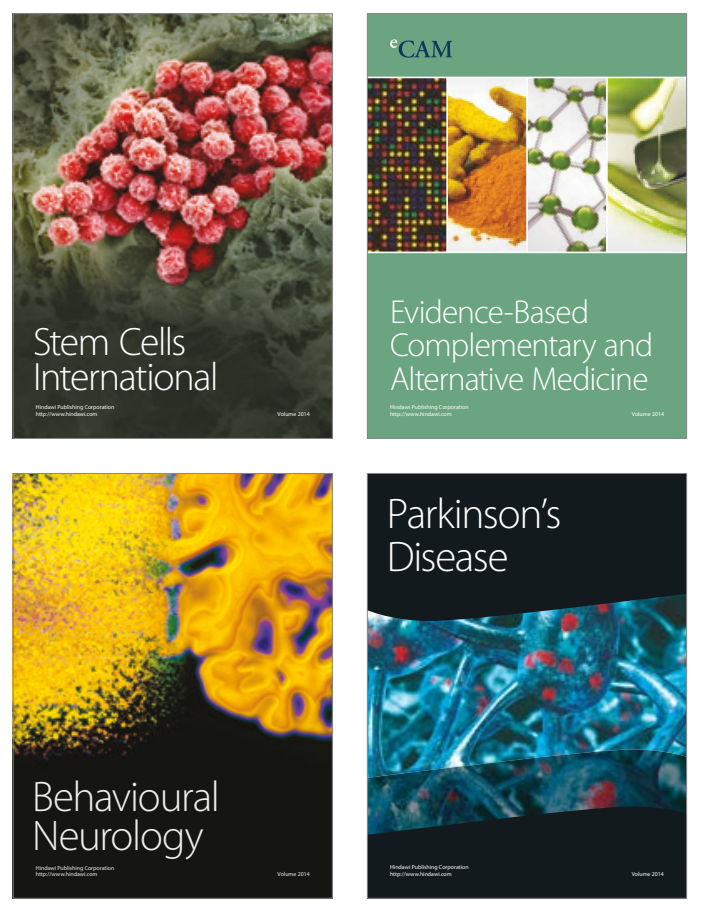
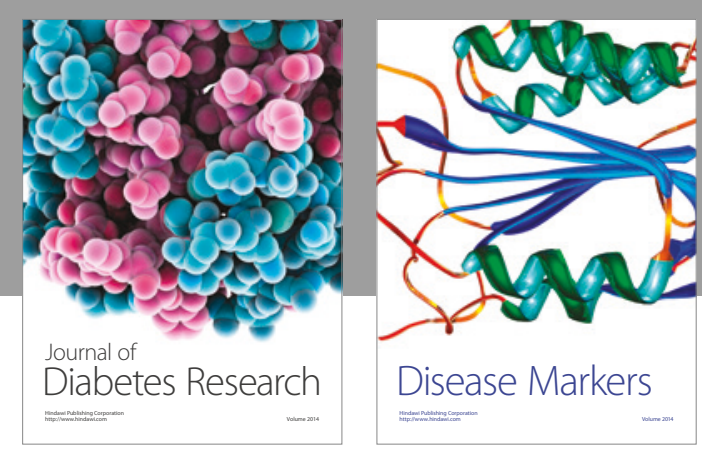

Disease Markers
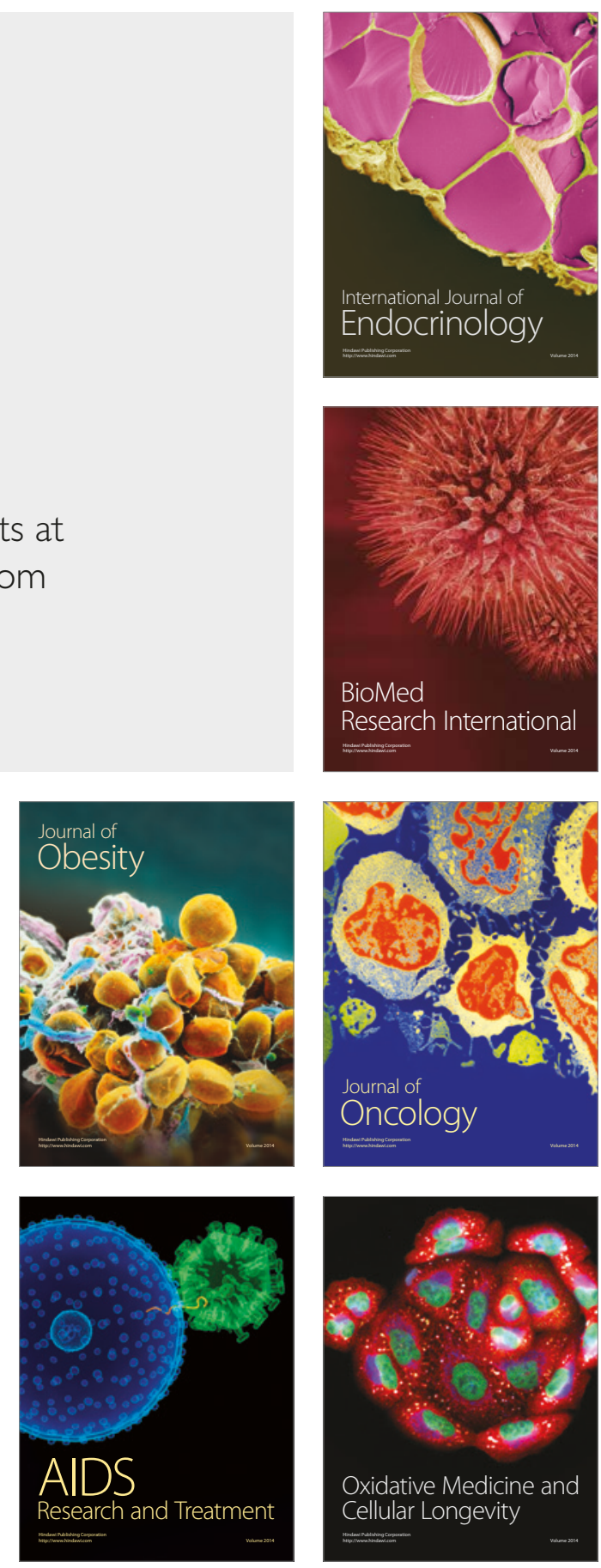